\title{
Breakfast Education Sessions - A novel approach to learning
}

\author{
Ian Brennan ${ }^{1}$, Dr Dermot Doherty², Anne McCabe²
}

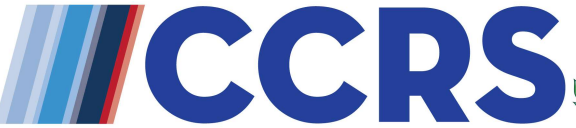

Critical Care \& Retrieval Services

${ }^{1}$ National Ambulance Service, ${ }^{2}$ NAS Critical Care \& Retrieval Services

Introduction \& Aims

The need for continuing professional development (CPD) is well recognised and is supported by professional bodies in all healthcare disciplines. It can be difficult to access CPD for those who work shift. Our aim was to create a multidisciplinary learning environment, within a National Ambulance Service (NAS) Station, and describe the participants responses to the education sessions

\section{Methods}

The National Transport Medicine Programme (NTMP) has recently become a service within the National Ambulance Service, under the new name of the NAS Critical Care \& Retrieval Services (NASCCRS). This service is responsible for transporting critically ill neonates, children \& adults. The Breakfast Education Sessions were planned with the purpose of creating a multidisciplinary learning environment and access to CPD. The sessions lasted for 1 hour from 730am-830am once a month. The sessions often focused on topics that the teams encountered during their clinical work. The sessions were also started to further enhance and build the multidisciplinary team combining of the NAS staff and the NASCCRS medical teams. The participants were sent a questionnaire to assess their attitudes towards the sessions.

\section{Results}

The majority of the participants $(78 \%)$ were very satisfied with the sessions, they thought they were educational, useful and inclusive. All of the participants believed it was a very well or extremely well way of building a multidisciplinary team, and $67 \%$ will use these sessions for their CPD. All free text comments were positive and encouraged more of these type of sessions.

\section{Conclusions}

The Breakfast Education Sessions, created by the team at the National Ambulance Service Critical Care \& Retrieval Services are a novel idea to learn, build teams and facilitate CPD.

We plan to extend the sessions with a view to streaming them in the future.

1: Knox, S., Cullen, W., \& Dunne, C. (2014). Continuous Professional Competence (CPC) for Irish paramedics and advanced paramedics: a national study. BMC medical education, 14(1), 41.

2: Knox, S., Cullen, W., \& Dunne, C. P. (2015). A national study of Continuous Professional Competence (CPC) amongst pre-hospital practitioners. BMC health services research, 15(1), 532.

3: PHECC CPC guide for EMTs available at www.phecit.ie 